\title{
The Importance of Plasma and Magnetic Investigations in Small Body Missions
}

A white paper for consideration to the Planetary Science and Astrobiology Decadal Survey 2023-2032

Michaela Villarreal ${ }^{1}$, Rob Lillis ${ }^{2}$, Janet G. Luhmann ${ }^{2}$, Christina O. Lee ${ }^{2}$, Joseph G. O’Rourke ${ }^{3}$, Rona Oran ${ }^{4}$, Kimberly M. Moore ${ }^{5}$, and Carol A. Raymond ${ }^{1}$

1. Jet Propulsion Laboratory, California Institute of Technology, Pasadena, CA

2. Space Sciences Laboratory, UC Berkeley, Berkeley, CA

3. School of Earth and Space Exploration, Arizona State University, Tempe, AZ

4. Department of Earth, Atmospheric, and Planetary Sciences, Massachusetts Institute of Technology, Cambridge, MA

5. Division of Geological \& Planetary Sciences, California Institute of Technology, Pasadena, CA

Mickey.n.villarreal@jpl.nasa.gov 626-590-8182

$$
\begin{aligned}
& \text { Seul-Min Baek }^{1} \\
& \text { Corey Cochrane }^{2} \\
& \text { Chuanfei Dong }^{3} \\
& \text { Robert W. Ebert } \\
& \text { Matthew Fillingim } \\
& \text { Amara Graps } \\
& \text { Am }^{6} \\
& \text { Emily Hawkins }^{7} \\
& \text { Bryana Henderson } \\
& \text { Zhengwei Hu }
\end{aligned}
$$

\section{Co-Signers:}

Jamie Jasinski ${ }^{2}$

Devanshu Jha ${ }^{9}$

Krishan Khurana ${ }^{10}$

Jiang Liu ${ }^{10}$

Rachel Maxwell ${ }^{11}$

Neil Murphy ${ }^{2}$

Tom Nordheim ${ }^{2}$

Lynnae Quick $^{12}$

Christopher Russel1 ${ }^{10}$

\author{
James Slavin ${ }^{13}$ \\ Beck E. Strauss ${ }^{12,14}$ \\ Marshall Styczinski ${ }^{15}$ \\ Neal Turrner ${ }^{2}$ \\ Dany Waller ${ }^{16}$ \\ $\mathrm{Xu}$ Wang $^{17}$ \\ Benjamin Weiss ${ }^{18}$
}

Co-signer Affiliations: (1) Korean Astronomy and Space Science Institute; (2) Jet Propulsion Laboratory, California Institute of Technology; (3) Princeton University; (4) Southwest Research Institute; (5) Space Sciences Laboratory, UC Berkeley; (6) Planetary Science Institute; (7) Loyola Marymount University; (8) XNano Science Inc.; (9) MVJ College of Engineering, India; (10) University of California, Los Angeles; (11) University of California, Santa Cruz; (12) NASA Goddard Space Flight Center; (13) University of Michigan; (14) National Institute of Standards and Technology; (15) University of Washington; (16) John Hopkins University; (17) University of Colorado-Boulder; (18) Massachusetts Institute of Technology

Acknowledgements: Michaela Villarreal is funded by an appointment to the NASA Postdoctoral Program at the Jet Propulsion Laboratory, California Institute of Technology, administered by Universities Space Research Association under a contract with NASA.

(C) 2020. All rights reserved. 


\section{Executive Summary}

The past decade saw a revolution in understanding a range of smaller bodies in the solar system, including asteroids, comets, and dwarf planets, with visits to Vesta, Ceres, Pluto, Charon, Ryugu, Bennu, and comet 67P Churyumov-Gerasimenko. Although these missions have greatly improved understanding of these bodies, science opportunities were missed due to the absence of plasma and/or magnetometer instrumentation. The exception to this is Rosetta to Comet 67P which carried both plasma and magnetic instruments that allowed for characterization of the plasma and magnetic environment relative to its outgassing rate. Understanding the evolution of the early solar system and its current water distribution are fundamental questions that can be addressed by these instruments. Magnetometer investigations provide information on a planet's internal evolution and dynamics by constraining its internal composition/layering (i.e. planetary cores and subsurface oceans) and detecting remanent magnetization. Plasma investigations can provide context for space weathering and detect escaping ions sourced from a neutral exosphere; this would be particularly beneficial if the next decade includes visits to outgassing asteroids. In addition, due to the limited interaction between the solar wind and small bodies, these instruments can also double as solar wind monitors. Thus, incorporation of these instruments serves a dual purpose to meet the goals of both NASA's Planetary Science and Heliophysics Divisions. Therefore, we recommend NASA's Planetary Science Decadal Survey emphasize the need to regularly incorporate plasma and magnetic investigations on all planetary mission payloads (including small bodies) and create a ride-along program which would provide an outlet to propose these instruments to perform interdivisional science.

\section{Introduction}

In situ studies of small bodies reveal important clues to the history and early formation of the solar system, a prime objective of NASA's Planetary Science Division (PSD). Instrument suites for small body missions often consist of cameras, ultraviolet, visible and infrared spectrometers, thermal imagers, and gamma ray and neutron detectors to classify the mineralogy, geology, and physical characteristics of their planetary surfaces. However, valuable information about a body's internal structure and atmosphere can also be gained by incorporating magnetometers, space plasma, and energetic particle instrumentation into the payload. While these types of instruments are typically solicited for planetary missions to bodies with magnetospheres and/or substantial atmospheres, they are seldom used in missions to small bodies.

The limited spatial extent of the interaction between small bodies and the solar wind would also allow these plasma and magnetic instruments to serve as solar wind monitors. This provides context not only for surface interactions that occur due to the bombardment of solar wind particles, but also for the propagation of solar events. The evolution and dynamics of the heliospheric plasma environment throughout the solar system is a central concern of NASA's Heliophysics Division (HPD), both for understanding fundamental plasma physics processes and for providing the scientific understanding that underpins space weather hazard forecasting for the Earth and space exploration. In this white paper, we call attention to the synergy between planetary and heliospheric science that would occur by the inclusion of plasma and magnetic investigations in all planetary missions, including those to smaller bodies.

Magnetic and plasma investigations onboard small body missions can provide insight into the target body's internal structure and composition, planetary magnetism, atmospheric production and composition, and surface-plasma interactions while also acting as solar wind monitors. Consistent incorporation of these investigations in small body missions (i.e. main belt asteroids, 
comets, trojan asteroids, dwarf planets, centaurs, Kuiper belt objects) would support NASA's science goals to 1) understand how solar system objects formed and evolved and 2) develop the capability to detect and predict extreme conditions in space (NASA 2014 Science Plan). We encourage the next decadal to advocate that these instruments be more widely integrated into small body missions.

2. Magnetic Investigations Constrain Internal Structure, Composition, and Evolution

Understanding the internal structures and compositions of small bodies is crucial for understanding the evolution of the early solar system. For example, it is still not well understood how pebbles and dust accumulate to eventually form planetesimals and planets. Studying how asteroids formed and evolved, both physically and chemically, would provide insight into this intermediary phase.

One method of studying the interior properties of a small body is through electromagnetic sounding. A conductor in the presence of a time-varying magnetic field will produce eddy currents on its surface, giving rise to an induced magnetic field which opposes the time-variation of the ambient field (Fig. 1a). Perturbations to the primary field caused by this induced magnetic field (Fig. 1b) can be used to determine the electrical conductivity of the body, which will depend on its composition. An example is the radius of the Moon's core, which was inferred using Apollo mission data by analyzing the induction response of the Moon to changes in the magnetic field as it passed through Earth's magnetotail [2]. Similarly, perturbations to the Jovian magnetic field as the Galileo spacecraft encountered Europa and Callisto provided evidence for the presence of subsurface, salty oceans [3]. Results from electromagnetic sounding joint with gravity measurements can be used to establish the body's conductivity profile and density structure.

Figure 1. (a) Induced magnetic field of a conductor opposes the time variation of the primary field. b) Observed perturbation to the primary field. Constraints on the body's composition and internal structure can be inferred by measuring its perturbation to the primary field. Figure from Khurana et al. (2009) [1].

(a)

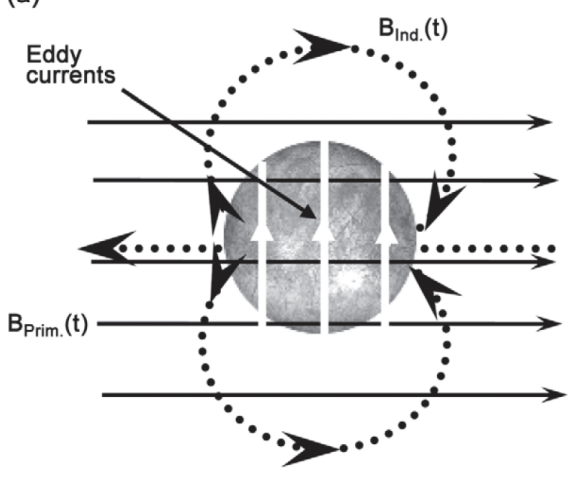

(b)

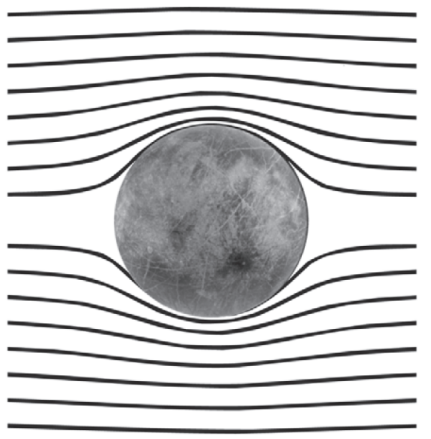

A body's internal evolutionary history can be inferred by detecting remanent magnetic fields, which can arise from two sources. First, thermoremanent magnetism in a secondary crust can indicate a past dynamo. The local magnetic field at the time that the crust formed is recorded by the electron spins of the ferromagnetic grains which align with the magnetic field as the rock cools below the Curie temperature of the minerals and becomes frozen-in; hence, after the dynamo ceases, that record may still exist within the crust, unless it has been destroyed by impact heating or chemical alteration of the magnetic carriers. Since a planetary body needs a convecting, electrically conducting fluid to generate its own magnetic field, detection of a past dynamo informs interior dynamics that occurred as the body evolved. Second, a small body can acquire remanent magnetism due to solar nebula magnetic fields if it formed and was cooled or aqueously altered before gas dissipated from the protoplanetary disk ( $<4 \mathrm{Myr}$ after CAIs) [4]. If remanent 
magnetization is detected at a body, its degree of differentiation could be used to distinguish which of these sources most likely provided the magnetization.

Remanent magnetization can be mapped using magnetometers, ideally in conjunction with electron reflectometers: magnetometers provide field measurements at the spacecraft altitude while electron reflectometers provide the ratio of the surface field to that at the spacecraft location by measuring electrons that are magnetically mirrored (reflected) closer to the surface. Crustal field mapping can still be performed with a magnetometer alone, albeit at a coarser resolution in field strength and spatial accuracy. This technique has been applied to map fields near the surface of the Moon [5], which confirmed it once possessed a dynamo. The upcoming Psyche mission, which will journey to asteroid (16) Psyche, will carry a magnetometer to determine if that body is a fragment of a planetesimal core by searching for remanent magnetization as a record of a past dynamo [6]. Future missions could also search for remanent magnetization of large C-type asteroids to determine whether they formed in the presence of nebular gas as predicted by pebble accretion.

Magnetometers enhance our understanding of planetary interiors through 1) electromagnetic sounding which provides constraints on the internal layering and composition of the body and 2) identification of planetary magnetism which informs the formation and evolution of the interior.

\section{Investigations Inform Surface Processes and Atmospheric Production}

Space weathering governs the surface evolution on small bodies, and is thus a major focus of remote-sensing investigations. Space weathering due to the solar wind occurs in two flavors: implantation and sputtering. Solar wind ion implantation leads to the creation of nanophase iron near the surface and causes a "reddening" in the spectral slope. The steepness of the reddened spectral slope in visible and infrared spectrometer data is used to estimate the length of exposure to solar irradiation to determine relative surface ages, and place bounds on absolute ages per spectral type. These calculations are based on laboratory studies which are then scaled to expected fluxes of the solar wind. Solar wind sputtering is a process whereby ions impacting the surface cause a cascade of collisions which result in the ejection of molecules from the surface material. Sputtering rates depend on the mass and energy of the impacting particle and hence differential fluxes are needed to accurately calculate these rates. Measurements of how different ion populations in the solar wind vary will improve calculations for space weathering rates, particularly at higher energies which are more variable and can penetrate deeper into the surface. Plasma instruments can provide these measurements in-situ.

Though small bodies are generally airless, they can produce time-varying exospheres through sublimation and/or sputtering if these processes are efficient enough. Neutrals that are sublimated or sputtered can become ionized and picked up by the solar wind. These exospheric ions can be observed directly with plasma instruments or indirectly through plasma waves contained in magnetometer data. The frequencies of plasma waves generated by pick-up ions are tied to the mass of the ion, thus providing information on the constituents present in the exosphere. If the pick-up rate is sufficiently large, it can mass load the solar wind, slowing it enough to produce a bow shock. Plasma instruments are best suited to infer outgassing rates [7], however detection of such a shock by the magnetometer could provide constraints on outgassing rates in their absence. The Rosetta mission observed this interaction at Comet 67P : the radius of the comet's diamagnetic cavity was shown to depend on the long-term temporal variation of the Comet's outgassing rate (Fig. 2) [8]. Methods to estimate outgassing rates using ion-cyclotron waves that are generated by the pick-up of exospheric ions have also been proposed [10]. 


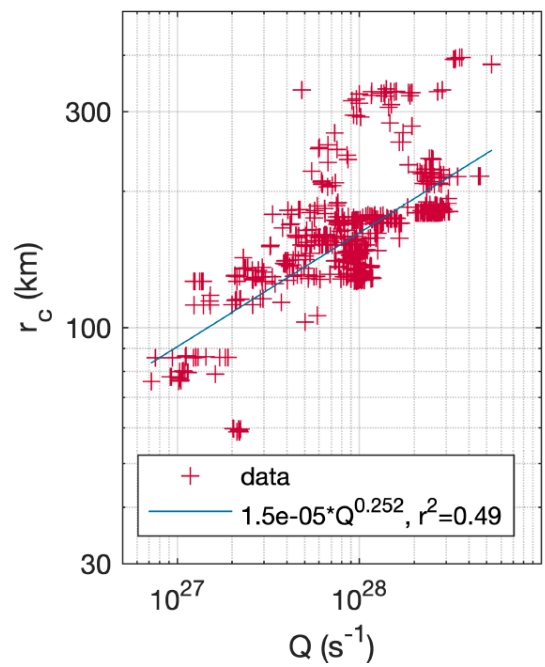

Figure 2. The radius of the diamagnetic cavity ( $\left.r_{c}\right)$ detected by Rosetta's Magnetometer at Comet 67P was found to be a function of its long-term gas production rate of water (Q). Figure from Goetz (2019) [9].

Water ice reservoirs on small bodies have become of growing interest to the scientific community. Determining the water content on asteroids and comets is essential to identifying reservoirs which could have provided water to the early earth and its neighboring terrestrial planets. The C-Complex asteroid group makes up two thirds of the asteroid belt by mass [11] with its members often showing signatures of hydrated minerals and/or water ice [12]. In recent years, telescopic campaigns have also observed several of its members to be actively outgassing [13]. Future missions to these types of bodies would greatly benefit by utilizing the methods described above. Water-ice has a low binding energy making it highly susceptible to solar wind sputtering and insitu plasma measurements would aid calculations of the vapor production that can be attributed to this process. Similarly, measurements of the outgassing rate through magnetometer data would inform the loss rate of water-ice and whether these reservoirs would require a source of replenishment. Thus, measurements of the heliospheric conditions experienced by small bodies would allow the determination of current water loss rates and inform water retention over time.

Plasma investigations can enhance our understanding of surface interactions by providing direct measurements for the fluxes of different ion populations impacting the surface which are used to calculate space weathering rates. They can also directly sample exospheric ions to infer outgassing rates. Magnetic investigations can indirectly probe exospheres by 1) detecting plasma waves created from pick-up ions which provide information on the masses of exospheric species and 2) detecting the solar wind-interaction (i.e. bow shocks) which can be used to infer outgassing rates.

\section{Investigations Inform Interplanetary Space Weather}

Distributed, multi-point measurements for a range of solar longitudes and heliocentric distances are needed to provide a 3-dimensional picture of how solar events, such as interplanetary coronal mass ejections (CMEs) and their leading shocks, evolve as they travel through the heliosphere. However, a majority of solar and heliospheric space weather monitors are located at a radial distance $\sim 1$ AU from the Sun. Data obtained at distances $>1$ AU would provide additional and important context for the interpretation of space weather observations. These data can be used to validate space weather models which are used for forecasting potentially hazardous events.

Measurements between $1 \mathrm{AU}$ and $4 \mathrm{AU}$, the region where most comets and asteroids can be examined in situ (Fig. 3), could 'flesh out' our understanding of this unique interplanetary plasma region where solar wind stream interaction regions (SIRs) steepen into dynamically-important 
compressions. SIRs modify the outer heliosphere solar wind properties, including CME-related disturbances, and act as barriers to CME shock-accelerated energetic particles. In addition, this region experiences solar wind mass loading due to microscale debris from asteroid collisions, increase in pick-up ion fluxes due to surface sputtering, and heavy ion production due to cosmic rays. Thus, the heliosphere from $1 \mathrm{AU}$ to $4 \mathrm{AU}$ is of great interest from many heliospheric as well as planetary research and space exploration perspectives.

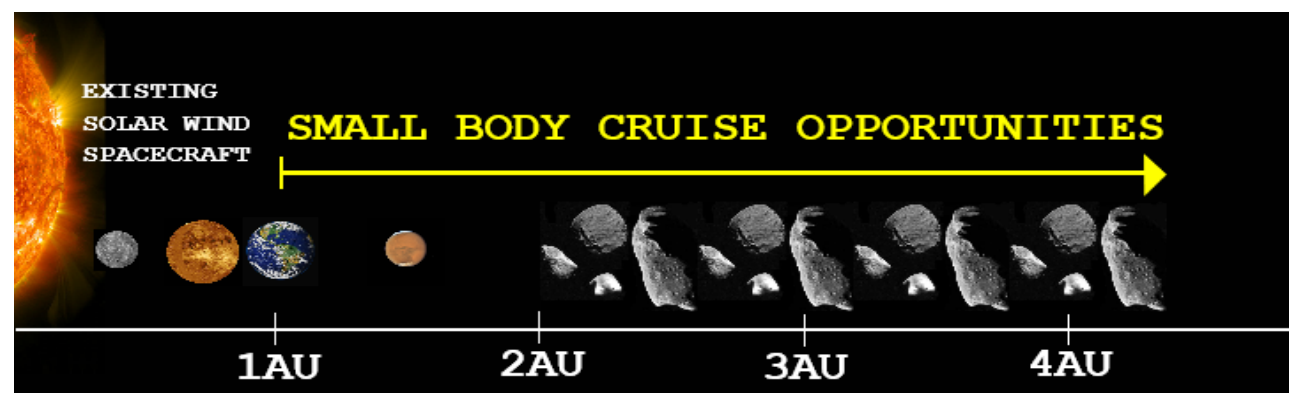

Fig 3. Solar monitoring spacecraft currently reside within $1 \mathrm{AU}$. Small body missions could provide a larger sampling of the heliosphere with plasma and magnetic instrumentation.

Planetary missions such as Voyager 1 \& 2, Cassini, Juno, and New Horizons have provided invaluable solar wind and interplanetary magnetic field (IMF) measurements as they travelled to the outer heliosphere $[14,15]$. However, the sampling by these missions of the heliosphere, a huge ( 100 AU) and highly dynamic environment, cannot replace a more continuous strategy for multipoint monitoring. Consistent measurements could be obtained using active spacecraft en route to or stationed at targets in the 1-4 AU region.

A recent example of such synergy occurred in connection with the Dawn mission. Though the Dawn spacecraft was not equipped with heliospheric instrumentation, its gamma ray and neutron detector (GRaND) was sensitive to energetic electrons and protons [16, 17], allowing it to detect the presence of solar energetic particle events while it encountered Vesta and Ceres. This allowed for a qualitative comparison of events detected at STEREO, Earth, Mars, and Dawn [18] and provided a 360-view of multiple event propagations. Though the GRaND instrument is not suited for quantitative measurements of the solar wind, it highlights the valuable science that can be done when monitoring the 1-4 AU region.

Plasma and magnetic investigations on probes visiting small bodies, or transiting the solar system, allow for the continuous monitoring of the solar wind past $1 \mathrm{AU}$. This monitoring would fill current knowledge gaps regarding physical processes and propagation of solar events which ultimately feed into space weather forecasting.

\section{Missed Science Opportunities on Small Body Missions in the Past Decade}

The scientific impact due to the absence of magnetometers and plasma suites onboard recent smallbody missions has already been felt by the research community. The past decade included missions to Vesta, Ceres, Pluto, Bennu, Ryugu, and Comet 67P. Of these missions, only the Rosetta Mission to Comet 67P carried both a magnetometer and a plasma suite. Meteoritic evidence has indicated Vesta, the Dawn mission's first target body, may have remanent crustal fields [19]. While Dawn was able to establish that Vesta's gravity field is consistent with a fully differentiated body with an iron core, whether this core ever generated a dynamo is a fundamental question that went unanswered. This detection would have confirmed Vesta to be the smallest known planetary body able to sustain a dynamo. Dwarf planet Ceres, Dawn's second target, has been shown to have a 
time-varying water exosphere as seen by telescopic campaigns [20]. However, despite Dawn's three-year rendezvous, no in-situ information could be gained about its exosphere with the exception of energetic electrons detected by GRaND which have been proposed to be a by-product of a temporary bow shock caused by outgassing [17,21]. The sources, production mechanisms, and lifetime for Ceres' exosphere still remain a topic of much debate. Though the New Horizons mission carried plasma instruments, the extent of the solar wind interaction with Pluto could not be fully captured without a magnetometer [22]. Additionally, observations of Sputnik Planitia have suggested a possible subsurface ocean which may still exist today [23]; this could potentially be investigated further with an electromagnetic sounding campaign on a future Pluto mission. While such instrumentation may sometimes be initially selected as part of a small body science payload, they regrettably have a history of cancellation.

The past decade of planetary science missed opportunities to verify an ancient dynamo at Vesta, characterize Ceres' exospheric production, and to fully capture Pluto's solar wind-interaction due to the absence of magnetometers and/or plasma instruments on their respective missions.

\section{How to Incorporate Magnetic and Plasma Investigations in Small Body Missions}

Missions to bodies in the main asteroid belt and beyond spend years en route to their destinations. We can take advantage of this cruise phase by the addition of small plasma and magnetic suites to the payload that are designed to monitor solar wind and IMF conditions. For Psyche-type missions, its cruise phase would allow $\sim 3$ years of continuous interplanetary measurements before arriving at the asteroid. Upon arrival at the body, these instruments can be further used to better understand both the body and its solar wind interaction.

Plasma and magnetic suite instrument packages would be relatively simple to incorporate into the design of future spacecraft missions due to their modest power and data requirements (Table 1). A simple solar wind monitoring package comprised of a gradiometer magnetometer on a modest boom,

Table 1: Plasma package resources for small body missions are modest.

\begin{tabular}{|l|l|l|l|}
\hline Instrument & Mass & Power & Data \\
\hline Magnetometer \& boom [24] & $1.3 \mathrm{~kg}$ & $0.8 \mathrm{~W}$ & $30 \mathrm{bps}$ \\
\hline Ion energy/angle/mass [25] & $3.3 \mathrm{~kg}$ & $4.2 \mathrm{~W}$ & $200 \mathrm{bps}$ \\
\hline Electron energy/angle [25] & $1.8 \mathrm{~kg}$ & $1.6 \mathrm{~W}$ & $40 \mathrm{bps}$ \\
\hline Energetic particle detector [26] & $0.9 \mathrm{~kg}$ & $3.2 \mathrm{~W}$ & $10 \mathrm{bps}$ \\
\hline Total & $7.3 \mathrm{~kg}$ & $9.8 \mathrm{~W}$ & $280 \mathrm{bps}$ \\
\hline
\end{tabular}
together with plasma and energetic particle detectors (mounted facing the sun and Parker spiral), are TRL 9 and would require only a few hundred bits per second, $\sim 10 \mathrm{~W}$ of power and $\sim 7 \mathrm{~kg}$ of mass (the latter two numbers will continue to decrease with further miniaturization). Though their physical requirements are minimal, such instrument suites face significant programmatic hurdles. Currently, the logistics of combining heliophysics and planetary science experiments on a mission is complicated by competing inter-divisional programmatic priorities and a lack of explicit opportunities for an interdisciplinary mission. In particular, there is no program or vehicle that prioritizes a science payload that is relevant to both divisions nor are there routine rideshare opportunities for HPD-relevant payloads (i.e. plasma instrumentation) either on already-selected PSD missions or as Science Enhancement Options (SEOs) as a part of mission proposals to PSD.

The recent selection of the ESCAPADE SIMPLEx mission for Phase A/B funding by HPD is a step in the direction of breaking down such logistical barriers. For example, the ESCAPADE mission focuses on plasma and field instrumentation. This accommodates researchers from heliophysics working in space weather and plasma interactions areas, but is also key to Mars science objectives (e.g. climate evolution) and space environment planning for human exploration. Mission opportunities are infrequent enough that full advantage should be taken of their potential 
for interdisciplinary science return, a concept that has held great advantages in the past for 'ridealong' science such as the Gamma Burst Detector on Pioneer Venus Orbiter.

One potential approach in this era of satellite miniaturization is the encouragement of technical development leading to suites of space environment instrumentation specifically designed for 'ride-along' purposes. Such a package might be viewed in mission development as an engineering unit for monitoring the spacecraft space environment - but at the same time produce valuable heliophysics and space plasma interaction data that would need to be accommodated in the housekeeping and/or science telemetry stream. If such units were routinely dispersed throughout the solar system on all SMD deep space missions, this would form evolving constellations of space environment observers whose information could be mined in routine ways as the mission sets evolved. In such an event, the matter of space environment conditions would automatically be a regular part of all missions' research and operations activities, benefitting interests across the board.

We suggest a ride-along program be established which would provide a channel for plasma and magnetic instruments to be proposed to perform interdivisional and interdisciplinary science.

\section{Summary}

Instruments typically used to monitor interplanetary space (magnetometers and plasma spectrometers) are also powerful tools that provide information on a body's internal structure, magnetism, surface properties, and atmosphere. Hence, the results returned by these instruments have both interdisciplinary and interdivisional scientific value. Though some previous small body missions did include plasma and/or magnetic investigations, these were the exception and not the norm. Looking forward to the next decade of planetary exploration, more emphasis on the inclusion of these instruments on small body payloads would greatly advance our understanding of both small body and heliospheric science.

\section{References}

[1] Khurana, K. K. et al. (2009), in Europa, University of Arizona Press, Tucson. [2] Russell, C. T. et al. (1981), Proc. Lunar Planet. Sci., 12B, 831-836. [3] Kivelson, M. G. et al. (1999), JGR, Vol. 104, No. A3, 4609-4625. [4] Fu, R.R. et al. (2014), Science, Vol 346, Iss. 6213, 1089-1092. [5] Lin, R.P. (1979), Proc. Lunar Sci. Conf. 10, 2259-2264. [6] Elkins-Tanton, L. T. et al. (2020), JGR: Planets, 125, e2019JE006296. [7] Wedlund et al. (2019), A\&A, 630, A37. [8] Goetz, C. et al. (2016), MNRAS 462, S459-S467. [9] Goetz, C. (2019), PhD Dissertation, Technische Universität Braunschweig. [10] Volwerk, M. et al. (2013), Ann. Geophys., 31, 2201-2206. [11] DeMeo, F. and B. Carry (2013), Icarus 226(1): 723-741. [12] Rivkin, A. S. et al. (2019), JGR: Planets 124: 1393-1409. [13] Hsieh, H. H. et al. (2018), ApJ, 155:96. [14] Burlaga, L. F. et al. (1982), JGR, Vol 87, No. A6, 4345-4353. [15] Gruesbeck, J.R. et al. (2017), GRL, 44, 5936-5942. [16] Prettyman, T. H. et al. (2011), S.S.R. 163, 371-459. [17] Russell, C.T. et al. (2016), Science, Vol 353, 1008. [18] Villarreal, M. N. et al. (2017), AGU Fall Meeting 2017, Abstract SH31B-1073. [19] Fu, R.R. et al. (2012), Science, Vol 338, Iss. 6104, 238-241. [20] Küppers, M. et al. (2014), Nature, 505, 525527. [21] Jia, Y.-D. et al. (2017), JGR: Space Physics, 122, 4976-4987. [22] Barnes, N. P. et al. (2019), JGR: Space Physics, 124, 1568-1581. [23] Nimmo, F. et al. (2016), Nature, Vol 540, 94-96. [24] Russell, C. T et al. (2016), SSR, 199, 189-256. [25] Kasper, J. C. et al. (2016), SSR, 204, 131-186. [26] Larson, D. et al. (2015), SSR, 195, 153-172. 